\title{
What Could Assistance Robots Learn from Assistance Dogs?
}

\author{
Márta Gácsi ${ }^{1}$, Sára Szakadát ${ }^{2}$, and Ádám Miklósi ${ }^{2}$ \\ ${ }^{1}$ MTA-ELTE Comparative Ethology Research Group \\ Pázmány Péter sétány 1/c, 1117, Budapest, Hungary \\ ${ }^{2}$ Department of Ethology, Eötvös Loránd University, \\ (marta.gacsi, szurtrikk, amiklosi62) @gmail.com
}

\begin{abstract}
These studies are part of our broader project that aims at revealing relevant aspects of human-dog interactions, which could help to develop and test robot social behaviour. We suggest that the cooperation between assistance dogs and their disabled owners could serve as a model to design successful assistance robot-human interactions.

In Study 1, we analysed the behaviour of 32 assistance dog-owner dyads performing a fetch and carry task. In addition to important typical behaviours (attracting attention, eye-contact, comprehending pointing gestures), we found differences depending on how experienced the dyad was and whether the owner used a wheel chair or not.

In Study 2 we investigated the reactions of a subsample of dogs to unforeseen difficulties during a retrieving task. We revealed different types of communicative and displacement behaviours, and importantly, dogs showed a strong commitment to execute the insoluble task or at least their behaviours lent a "busy" appearance to them, which can attenuate the owners' disappointment. We suggest that assistant robots should communicate their inability to solve a problem using simple behaviours (non-verbal vocalisation, orientation alternation), and/or could show displacement behaviours rather than simply not performing the task.

In sum, we propose that assistant dogs' communicative behaviours and problem solving strategies could inspire the development of the relevant functions and social behaviours of assistance robots.
\end{abstract}

Keywords: assistance robot, dog behaviour model, ethological approach, social interaction

\section{Introduction}

Considering the aging western industrialized societies, in the near future it will be an absolute necessity that the elderly and disabled people could successfully communicate and cooperate with home assistance robots. There are many different endeavours to design technological aids helping the rehabilitation of the physically disabled, ranging from intelligent wheelchairs to different types of assistant robots 
(1). Most of these robots are designed for some specific roles and functions. Assistance systems are also developed, for example the small home robot, MamoruKun is able to inform his owner where an object is located communicating verbally or by pointing at the object, and it can also cooperate with his humanoid buddy and ask it to get the object for the user. This bigger robot is able to clean up rooms, manipulate dishes, open and close doors, do the laundry, and it even learns from its mistakes (2). Another example can be the mobile robotic assistant of the Nursebot project, Pearl. She has two functions: reminding the elderly about their routine activities and guiding them through their environments (3).

However, the commercialization of either type of assistance robots is planned only in ten years, partly because people usually find them disturbing and/or complicated to operate or communicate with. To make humans feel the interactions with the robot more natural, in addition to the technical aid it is essential for the robot to act in accordance with the given social context and to show relevant social abilities (4).

Based on the general assumption that people find a similar companion easier to deal with, the most common strategy both in scientific and commercial environment is to design humanoid robots that have (seemingly) humanlike features and capacities. One of the potential problems with the use of anthropomorphic behaviours lies in the controversy that humanoid robots are likely to raise the expectations of the user in terms of its capabilities and interactional affordances, but present-day robots are simply not able to fulfil these expectations (5). Recently an alternative suggestion has come up arguing for the use of non-human social animals as models for robot social behaviour: emphasizing the fact that human-animal interaction provides a rich source of knowledge for designing social robots that are able to interact with humans under a wide range of conditions (6). The idea that in addition to its technical help a robotic assistant could be a suitable companion directed the attention of researchers to use pets as potential behavioural models (7).

In case of assistant robots, the type of function and the limited abilities (compared to that of human assistants) suggest an asymmetrical social relationship between the human user and the robot, which is similar in many ways to the dog-owner relationship (8). Recently, scientific evidence has supported the idea that dog-human interactions provide a promising model system to study the emergence of social competence (9). Assistant dogs can successfully communicate and cooperate even with a disabled owner, and show social behaviours, such as attachment, which humans can easily understand without massive prior learning. This way, beside technical assistance, these trained dogs provide social support for their owners (10)

It has already been suggested that using service dog models to design subtle motor behaviours for the manipulation skills of assistance robots is a highly beneficial and cost effective method (11). We would go further and give dogs a more ambitious role: based on the arguments above we suggest that the social behaviour of dogs and specifically the owner-assistant dog interactions should be used as a model in developing robot companions.

As there were efforts to implement the ethological model of the dog to Sony's developmental robot, AIBO (12), we want to stress that our aim is not to develop a doggy robot. We do not believe that a robot that resembles a dog, for example AIBO, would be an ideal assistance robot. Rather, we propose the use of an embodiment optimally fitted for the specific function of the robot and applying specific 
behavioural models for different technical/social functions, such as service dogs for the disabled owners or hearing dogs for deaf people.

In order to do so we plan to create plenty of rich, relevant and realistic contexts for the interactions, in which we can observe and evaluate the joint activities of dogs and humans. After extracting the relevant set of behaviour elements based on the desired function, we can adapt the applicable ones to robots of different embodiments and capacities.

Our first attempt to support the dog-model idea applied one of the typical scenarios between service dogs and their disabled owners: when the dog helps the transportation of objects. In this study an appearance-constrained Pioneer robot used dog-inspired affective cues to communicate with its owner and a guest in a fetch and carry scenario. The findings suggested that even limited modalities for non-verbal expression (proxemics, body movement and orientation, camera orientation) offered by the robot were effective for developing/helping the communication (13). In the present paper we wanted to provide deeper insight into such interactions by detailed analyses of the behaviour of a large and diverse sample.

To develop a life-like and flexible behavioural set for assistance robots it is inevitable to prepare assistant robots for problem situations because it does not seem too pessimistic to assume that they will face several insoluble problems and cannot meet their owners' requirements while performing their tasks. When a 'machine' cannot fulfil our requirements, we are disappointed and annoyed. In contrast when a trained assistant dog cannot obey the owner's commands the owners seem to be rather indulgent and forgiving. We assume that dogs may show different types of communicative behaviours, such as vocalisation or gaze alternation to signal the problem to the owner. Moreover, they may try to perform some alternative activities that are connected to the original task to some extent. In addition, we expected the dogs to perform displacement behaviours, which can influence or even inhibit the negative feelings of the owners, similarly to "guilty" behaviour after transgression (14). Displacement behaviour emerges both in humans (15) and non-human animals (16) in conflicting situations. For instance, when dogs find themselves in a situation when they are unable to solve a task or a problem, they show typical out-of-context behaviours (e.g. mouth-licking, yawning, sniffing the ground, scratching the ground, pace up and down between the owner and the showed place), which reflect their confusion.

In this paper we observed the interaction of assistance dog-owner dyads in two studies. In Study 1, we investigated the types of behaviours and interactions that can be observed in dog-owner dyads when they perform a fetch and carry task. Moreover, we examined whether there are any difference in the above behaviours depending on how experienced the dyad was and whether the owner used a wheel chair or not. In Study 2, we observed a subsample of dogs in two different types of inhibited trials and described both their typical responses and individual differences when they encountered an unsolvable task commanded by the naïve owners. 


\section{Study 1 - Fetch and Carry}

The fetch and carry task may include picking up, carrying and placing objects at home or outside the house. This ability of the assistance dogs increases the owners' independence and allows a greater range of activities, because while assistive, still requires movement and actions on the part of the owner, thus increasing the amount of physical activity available to him rather than decreasing it.

In this study the dog was supposed to fetch and carry a basket from a start point to a given target place. An assistant capable of performing the task properly need to be able to: go to the person on command, understand human communicative cues to identify the target object, hold and carry the basket, follow or go with (beside) owner or go ahead (with continuous feedback for owner's orientation/instructions), put down the basket on command.

The only instruction for the owners was that they cannot touch the dog or the basket enabling us to reveal spontaneous cues and behaviours of the dyads during the interactions.

\subsection{Method}

\section{Subjects}

We observed 32 dog-owner dyads. All dogs were trained assistance dogs, either therapy dogs or assistance dogs for the disabled. They were tested with their owner (O). Half of the dyads were novice, with dogs having the same training as the other group, but not as much experience in various contexts. The other 16 dyads were experienced; these dogs had been working with their Os for years. In case of 8 dyads in both the novice and experienced group Os were wheel-chaired. All dogs were more than one year old. (Table 1 contains the data of the dogs.) The training of these dogs were based on the principle that they should be eager to please their owner, that is, they must do their best to find out the task the owner communicates and to cooperate in its execution, even if the human's communication is not completely clear.

Table 1, Independent data of the dog sample

\begin{tabular}{|c|c|c|}
\hline $\mathrm{N}=32$ & NOVICE & EXPERIENCED \\
\hline OWNER & $\begin{array}{l}4 \text { males, } 4 \text { females } \\
\text { Mean age: } 4 \text { yrs. } \\
\text { Breeds: Belgian sheep dog, sheltie, golden } \\
\text { retriever, border collie, Airedale, } 2 \text { lab mixes }\end{array}$ & $\begin{array}{l}3 \text { males, } 5 \text { females } \\
\text { Mean age: } 3.8 \text { yrs. } \\
\text { Breeds: } 3 \text { Belgian sheepdogs, } \\
\text { vizsla, lab mix, } 3 \text { mongrels }\end{array}$ \\
\hline $\begin{array}{l}\text { WHEEL- } \\
\text { CHAIRED } \\
\text { owner }\end{array}$ & $\begin{array}{l}5 \text { males, } 3 \text { females } \\
\text { Mean age: } 2.6 \text { yrs. } \\
\text { Breeds: Belgian sheep dog, golden retriever, } \\
\text { cavalier, cocker, poodle, lab mix, leonberger } \\
\text { mix, mongrel }\end{array}$ & $\begin{array}{l}5 \text { males, } 3 \text { females } \\
\text { Mean age: } 5.6 \text { yrs. } \\
\text { Breeds: } 3 \text { Belgian sheepdogs, } \\
\text { golden retrievers, collie mix, } \\
\text { lab mix }\end{array}$ \\
\hline
\end{tabular}




\section{Procedure}

The tests were conducted at a visually separated location in a park that was familiar to the dogs. The dog and $\mathrm{O}$ were positioned at the start point. The experimenter (E) placed the basket in front of them. The target place was positioned 10 metres from the start point and marked by three $80 \mathrm{~cm}$ long sticks forming an equilateral triangle. The behaviour of the dog was recorded from the side by E from a distance of 5-6 $\mathrm{m}$. The records were analysed later.
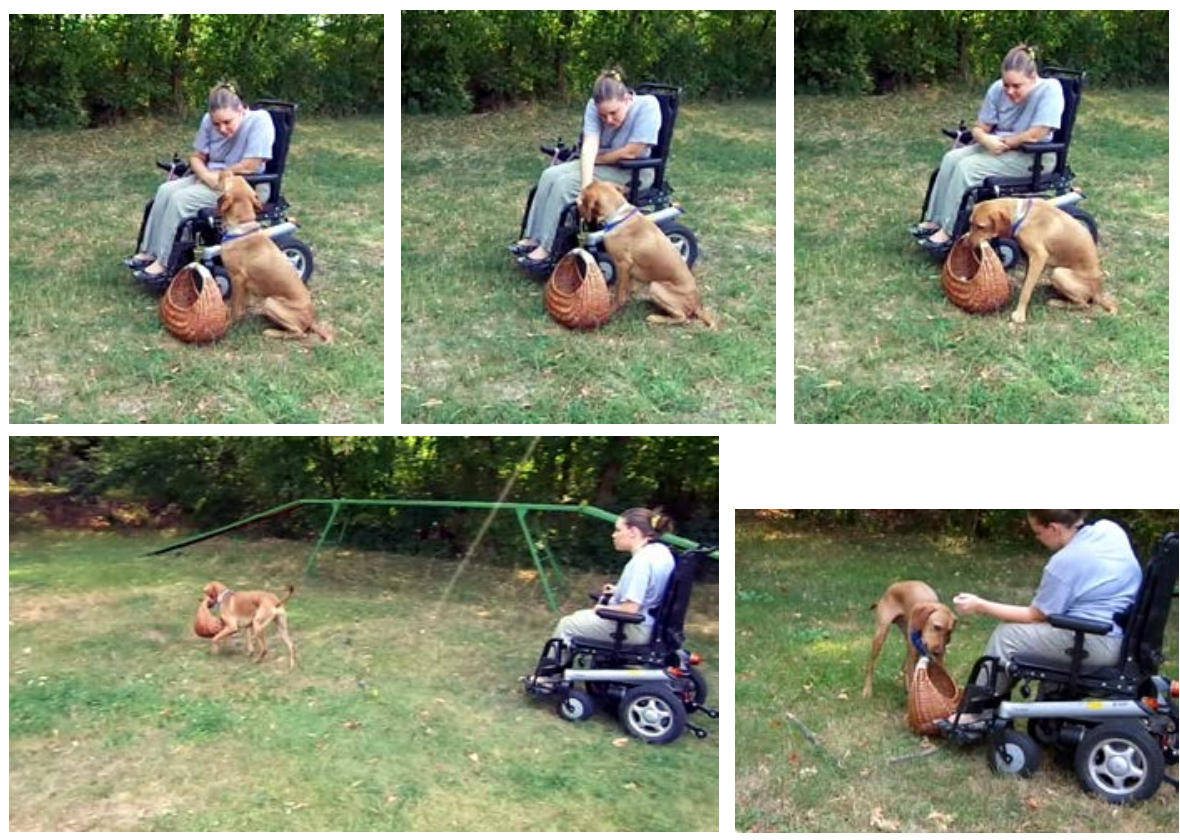

Fig.1. Behavioural sequence of the test: joint attention, pointing, picking up basket, carrying basket ahead of owner, placing basket at target area

\section{Data analysis}

During the analysis our main focus was on the communication between dog and $\mathrm{O}$ (paying and getting attention, communicating target object, target location and expected actions) and the dynamics of their movements (relative position of the dog while carrying the object). Since the duration of the whole task depended greatly on O's motor ability, the three main parts of the test; 1) picking up, 2) carrying, and 3) placing the basket, were analysed separately. The following variables were coded: joint attention (yes-no), number of verbal instructions, pointing gestures (yes-no), position of the dog (relative to the owner) when performing the carrying task (ahead beside - behind), duration of the picking up and placing tasks (s).

Multivariate analysis of variance was applied to compare durations and number of commands. Kruskal-Wallis test and Mann-Whitney test were used to compare the position of dogs in different groups. 


\subsection{Results}

\section{Types of verbal communications}

The owners' verbal instructions could be categorised as commands (verbs), name of dog, name of object (“basket”), praises (“well done”), and inhibitions (“no”, “don’t”) in all three parts of the test. The proportion of the categories during the entire test duration was as follows: commands (verbs) 63\%, name of dog $14 \%$, name of object $8 \%$, praises $13 \%$, and inhibition $2 \%$.

\section{Picking up (interaction initiation)}

We observed joint attention in case of all dog-owner dyads at the very beginning of the task. This means that the dog and O looked at each other before the dog got hold of the basket. In most cases eye-contact was spontaneous, that is, the dog oriented to $\mathrm{O}$ right after having been seated at the start point. If the dog oriented somewhere else $(\mathrm{N}=10), \mathrm{O}$ tried to attract its attention by calling its name before giving any instructions. After this, all but four Os pointed at the basket when communicating the task to the dog. Three of the exceptions were from the advanced/wheel-chaired group, where Os either needed both hands for driving the mechanical wheel chair, or could not move their arms. All four Os "pointed" toward the basket with their head.

The number of verbal instructions and the duration of the task showed significant correlation, and both varied according to the experience, because novice dogs needed more time and more verbal instructions to execute the task.

\section{Carrying}

Compared to the novice group, experienced dogs tended to carry the basket more frequently by going ahead of $\mathrm{O}$ than going behind. Using a wheel chair in itself did not make a general difference in this respect, however, post hoc tests revealed that in the experienced wheel-chaired group dogs had the tendency to carry the basket going ahead of their Os.

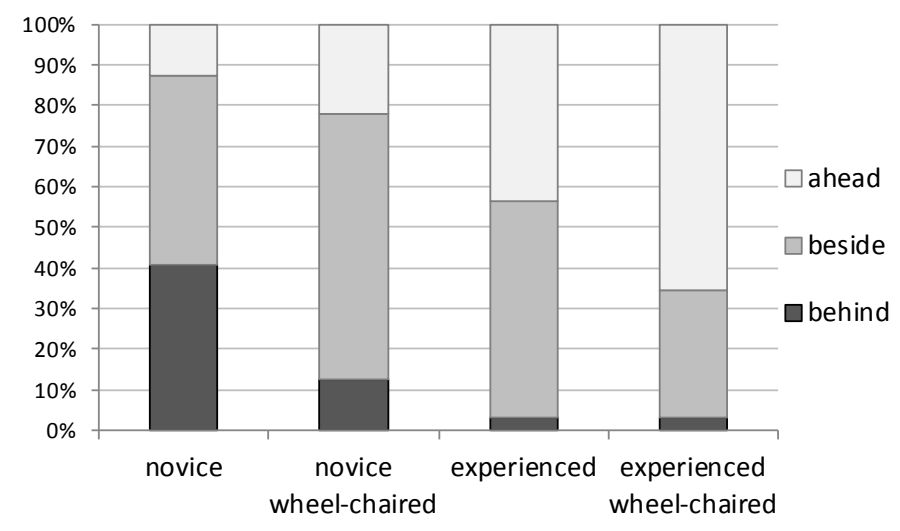

Fig. 2. Position of dogs during carrying: proportion of dogs that walked ahead, beside or behind the owner in the four groups 
Novice dogs needed more verbal instructions to fulfil the task. Although the carrying part of the test took more time for the wheel-chaired Os, they did not talk more to their dog.

\section{Placing}

The number of verbal instructions and the duration of the task showed significant correlation. We found significant effect of both the experience and if the owner was wheel-chaired or not. The significant interaction of these effects revealed that the novice wheel-chaired group needed much more time and instructions for successfully placing the basket than the other three groups.

\section{Table 2}

Number of dyads where joint attention and/or pointing gesture could be observed during the given task

\begin{tabular}{|l|c|c|c|c|c|c|}
\hline & \multicolumn{2}{|c|}{ Pick up } & \multicolumn{2}{c|}{ Carry } & \multicolumn{2}{c|}{ Place } \\
\hline & joint att & point & joint att & point & joint att & point \\
\hline Novice & 8 & 8 & 1 & 0 & 4 & 2 \\
\hline Novice - Wheel-chaired & 8 & 8 & 7 & 4 & 7 & 7 \\
\hline Advanced & 8 & 8 & 4 & 3 & 8 & 7 \\
\hline Advanced - Wheel-chaired & 8 & 5 & 6 & 2 & 4 & 5 \\
\hline
\end{tabular}

\section{Study 2 - Inhibited Tasks}

In this study after the dogs successfully performed a well-known and easy task several times, we presented them with an insoluble version of the same task. We assumed that dogs mainly would show two types of response to the situation. They would either react actively by trying to communicate with the owner (e.g. vocalizing, gaze alternation) (17), or passively, by showing displacement behaviour (e.g. lip licking, yawning, stretching). These behaviours may help the owner to realize that his/her request is ambiguous or not executable. Analysing dogs' responses in such scenarios can help to reveal how a robotic assistant should behave in such situations and/or how they should communicate a problem to the human.

\subsection{Method}

\section{Subjects}

A subsample of dyads was tested in this study: novice group $(\mathrm{N}=4)$, novice-wheelchaired group $(\mathrm{N}=4)$, experienced group $(\mathrm{N}=5)$, experienced-wheel-chaired group $(\mathrm{N}=6)$. 


\section{Procedure}

The two tests took place in an unfamiliar laboratory room at the Department of Ethology, ELTE, Budapest. The dogs entered the room together with $\mathrm{O}$ and were allowed to explore the test room for a few minutes before the test.

The two tests were both simple fetch and carry tasks and always followed each other in a fixed order. In test 1 , the task was insoluble because the basket was not at the place where it was supposed to be. In test 2, the dog encountered a noncooperative experimenter (E) who inhibited the fulfilment of the task by not handing over the basket. E who manipulated the basket was always a trained woman. The tests were video recorded and analysed later.

\section{Test 1}

There were two chairs inside: one in the middle for $\mathrm{O}$ and one in the corner for the dog's leash. The dog was sitting next to its $\mathrm{O}$ off leash. In the other end of the room there was a small cupboard and a barrier attached to it making one of the corners invisible for the dog and the owner. Opposite to the barrier there was an open door.

Prior to the test E explained the scenario shortly to $O$ and laid down two rules: (1) to use only the following 3 commands: "bring", "basket" or "come" (to avoid the use of commands such as "seek" or "find") and (2) after sending the dog to fetch the basket, they are not allowed to give further commands till the dog initiates eye contact with them.

Warm up phase

The test began with a basic basket retrieving task, which is a basic task for these dogs given that this is a cornerstone of their training (and an everyday activity for the experienced ones). First E showed the basket to the dog, put it down to the floor in the middle of the room about 2 metres from the dyad, closed the door and settled at the wall. The dog was commanded by $\mathrm{O}$ to retrieve the basket. Then in the two following trials after showing the basket to the dog it was hidden behind the folding, and the dog had to retrieve it from there when asked. After the completion of the task the dog always returned to its original position at the side of O. Next, the scenario was completed with a new element to prepare for the test trial: after hiding the basket $\mathrm{O}$ had to cover the dog's eyes and also close his/her own eyes. Non-visible to them $\mathrm{E}$ closed the door, stepped back to her place, and instructed the $\mathrm{O}$ to open her or his eyes, uncover the dog's eyes and execute the basket collecting task in the same way as before. This procedure was repeated two times.

Testing phase

The fifth trial was the inhibited one. When the eyes of the dog and $\mathrm{O}$ were closed, $\mathrm{E}$ removed the basket to the neighbouring room (far enough from the door, so the dog was not able to smell it from the experimental room), closed the door, returned to her usual position and instructed the owner to execute the same procedure as usual. The duration of the trial was 2 minutes measured by E from the moment that the dog was faced with the lack of the basket behind the cover. E stood still at her usual place. After 2 minutes E opened the door and gave the basket to the dog that returned it to $\mathrm{O}$ and was praised. 


\section{Test 2}

E showed the basket to the dog, sat down onto a chair placed about 2 metres from the dyad in the middle of the room, and instructed $\mathrm{O}$ to command the dog to bring back the basket. However, when the dog got hold of the basket E did not hand it over, but clung on it. The trial lasted for 1.5 minutes measured by $\mathrm{E}$ from the moment the dog discovered that E did not allow it to take away the basket. The same rules were applied for giving commands to the dog as in Test 1. After 1.5 minutes E released the basket, the dog could return it to $\mathrm{O}$ and was praised.

\section{Data analyses}

The analyses of these tests focused mainly on the behaviour description of the dogs in the specific situations.

The following variables were coded in Test 1: latency of looking at owner and experimenter (s), approaching owner (yes-no), vocalization (yes-no), displacement behaviours (yawn, stretch, paddle, scratch itself, lick its lip, shake) (yes-no), fetching other object (yes-no), duration of looking for basket in $1 \mathrm{~m}$ around cupboard (s) and at other places in the room (s).

The following variables were coded in Test 2: latency of looking at owner (s), looking at experimenter when not manipulating the basket (yes-no), approaching owner (yes-no), vocalization (yes-no), displacement behaviours (yawn, stretch, paddle, lick its lip, shake) (yes-no), fetching other object (yes-no), duration of pulling (s) and chewing (s) the basket.

Due to the relatively small number of dyads from the specific groups the data were mostly analysed as one sample. Multivariate analysis of variance was applied to compare durations of searching in different places across the groups.

\subsection{Results}

\section{Test 1}

All but one dogs looked at $\mathrm{O}$ at least once during the inhibited trial, the mean number of gazes were 4.2, and the mean latency of the first gazing was 32 seconds. A large proportion of dogs (63\%) also approached O, and 74\% of them even gazed at E. The latency for gazing $\mathrm{E}$ was bigger than that of gazing at O. Several dogs showed even more explicit forms of communication: $26 \%$ vocalised while confronting the problem.

Passive forms of expressing confusion could be also observed: $32 \%$ of the dogs exhibited displacement behaviours, and $26 \%$ fetched or manipulated some other object, mainly the leash or tiny pieces of the test set up (tape).

Moreover, dogs kept on looking for the basket on the average for $35 \mathrm{~s}$ around the cupboard and for $58 \mathrm{~s}$ at other places in the empty room. Dogs of wheel-chaired Os looked for the basket less at irrelevant places. 


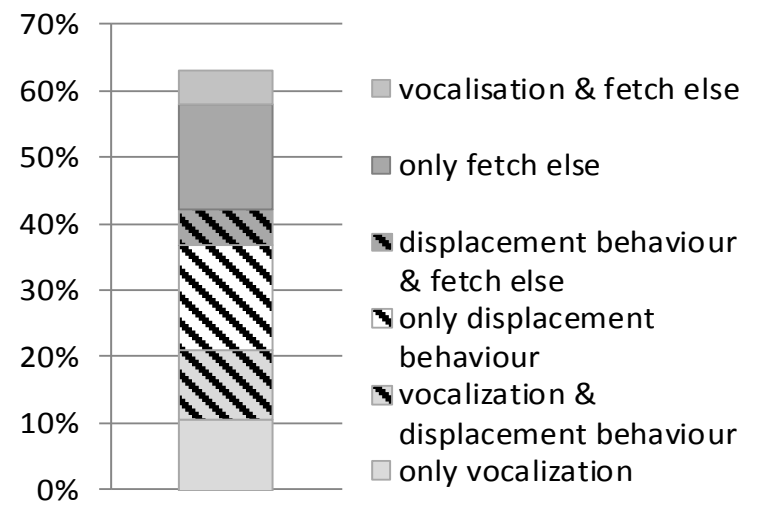

Fig. 3. Proportion of dogs that vocalised, exhibited displacement behaviours, fetched other objects or exhibited some combination of these behaviours when they could not find the object the owner asked for.

\section{Test 2}

When E did not allow them to take the basket, $63 \%$ of dogs looked at $\mathrm{O}$, the mean latency for gazing was $33 \mathrm{~s}$. Moreover, $32 \%$ of the dogs approached O, and $67 \%$ of them gazed at E. Many dogs (32\%) vocalised while facing the non-cooperative E, and a relatively large proportion of dogs (42\%) exhibited displacement behaviours.

Dogs tried to manipulate the basket in different ways. All dogs pulled the basket strongly; mean duration for pulling was $28 \mathrm{~s}$. All but 4 dogs also chewed the basket; the mean duration for chewing was $27 \mathrm{~s}$. The durations of pulling and chewing did not correlate with each other. In this test only two dogs retrieved another object to $\mathrm{O}$.

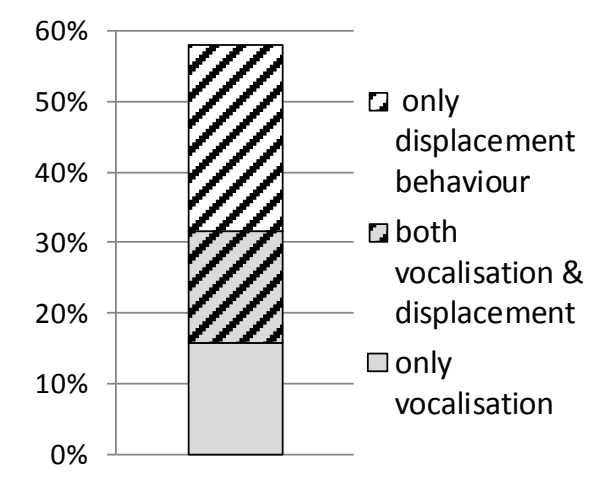

Fig. 4. Proportion of dogs that vocalised, exhibited displacement behaviours or did both when the experimenter did not hand over the object 


\section{Discussion}

These studies range from finding the plausible channels of communication in humanrobot-dog swarms applied for specific rescue purposes, through adapting the behavioural characteristics of dog-human attachment bond to robot-human dyads (18), to modelling exploratory behavior of assistance robots designed for home-care applications (19). Although nowadays mainly assistance dogs help the elderly and disabled humans in their every-day life, in ten years assistance robotics will certainly make a significant breakthrough in this field. In order to enhance the effectiveness and quality of the interaction between humans and robots, users should be able to interact with assistance robotic systems in a natural way. Presently most assistance robots try to mimic human capacities, embodiment and behaviour, even though neither their function nor their abilities make them comparable to a human assistant yet. We argued that the adaptation of some relevant behaviour patterns observed in assistance dog-owner interactions could improve the ability of assistance robots of different embodiments to provide successful social and physical assistance of elderly people in a home environment. In order to design such a robot we have to examine owner-dog interactions with a special focus on the situations the assistant robot will face during its duty.

Our observations of a fetch and carry task showed that even the crucial elements of the interaction, such as attention getting behaviours and the human partner's verbal communication, can show both context specific and dyad specific features (see for similar results 20.). The contribution of the simple verbal instructions to the successful execution of the different tasks (fetching, carrying, placing) needs further analyses, but importantly, during the interactions owners' verbal activity was complemented by non-verbal communication. This draws attention to the significance of the ability for parallel perception and processing of human verbal and non-verbal communication, for instance the need to be able to interpret gestures in assistance robots (see e.g. 21). We revealed that joint attention was required for the initiation of task execution. As a rule, owners draw their dog's attention before giving any instruction, and they look at each other before performing further actions. This implies that even very simple non-humanoid robots should show their "attentional state" by at least the orientation of their body and/or head to show "attention" and facilitate the initiation of the interaction.

Pointing at an object to be manipulated by the assistant also proved to be a typical action, and dogs were very successful in relying on this gesture. Convincing evidence proves that for this no special training is required because dogs are successful in spontaneous use of human pointing gesture (22). Dogs' ability to select the requested object from several objects placed close to each other need further investigation, but we suggest that the interpretation of simple pointing gestures (using extended vectors of the outstretched arm/pointing finger) could be a very useful skill for assistance robots and ongoing research (e.g. 23) should proceed forward in this area applying results from dog cognitive studies (e.g. 24).

During the carrying task the dogs seemed to apply different strategies depending on their experience. Novice dogs followed the owner toward the target area or walked beside them (25), however, experienced ones could use more subtle information to find out the correct direction, using the owner's pointing gesture or verbal 
instructions, or by extrapolating the direction of the initial movement of the owner to predict the location of the target. The more advanced performance of the wheelchaired group is important to note, because in real life situations the ability to navigate the assistant in front of them and not being forced to look back regularly could be especially useful for owners in wheel-chair. Although this particular activity (carrying) is mainly driven by the user's capabilities, because the movement speed of the user regulates the time needed for dog, wheel-chaired owners did not use more verbal instructions for this task. This implies that the number of commands is not simply linked to the duration of the task, but rather to the quality of the cooperation.

In addition to group level differences, we could observe marked within group (individual) differences during the interactions. Placing an object to a predefined place could be difficult for the novice wheel-chaired group because the usual end of the basket carrying task is to hand over the basket to the owner and some inexperienced dogs were not used to flexibly combine the learnt behaviour elements on command (that is, placing the object at the target point).

In Study 2 dogs faced two situations they might encounter in everyday activities when they are not able to perform the tasks they had been asked for. Both scenarios were based on the fetch and carry abilities of dogs, but the situations differed in a crucial element. In Test 1 , there was no object at the location indicated by the naïve owner. In Test 2, the object was there but the execution of the required action was actively inhibited by the experimenter. As expected, most dogs tried to express their confusion and negative inner state in at least one way. In contrast to Study 1, during the inhibited trials dogs displayed some specific vocalisation, that is, they emitted high pitched voices that could reflect their inner state in an explicit manner, which needs no training to interpret by humans (26).

There was also a tendency to use more passive forms of expressive behaviours by showing displacement behaviours. We must admit that exhibiting such behaviours cannot be regarded as communication of the ambiguity of the situation, and might only be effective if the owner is sensitive or experienced enough to read these unintentional behavioural signals. However, one of the approaches of social psychology $(27,28)$ sees the roots of human embarrassment in the appeasement behaviours of animals. These unintentional actions have appeasement-related functions, pacify partners in case of social transgressions by reducing aggression and evoking social approach in others, thus restoring the social interaction and relation. So the calming value of these behaviours - processed by the owners either consciously or unconsciously - can account for the difference in the reactions of humans to the failures of the dogs and robotic agents.

The major message of the results of the inhibited trials is that dogs do not give up easily if they face a "seemingly" insoluble task. Almost each dog showed a strong commitment to execute the task or at least the behavioural elements discussed above lent a "busy" appearance to them. This strong endeavour to fulfil the task can be an attractive characteristic in the eyes of the owners and can attenuate their disappointment. These behaviours (e.g. hesitantly turning around, moving around close to the place of the task to be executed, looking/moving back and forth between the user and the aimed object, emitting some high pitched sound) should be adapted to assistance robots to enhance their similarity to a living helper. Thus we could count on the empathy, understanding and forgiveness in humans that living beings are able 
to evoke in such situations. Obviously, we do not suggest that an assistance robot should whine, yawn or scratch itself when it is in an ambiguous situation. We propose that the problem solving strategies of assistant dogs could inspire the development of the relevant functions and social behaviours of assistance robots. We suggest that it would make HRI more fluent and less stressful if assistant robots could communicate their inability to solve a problem using simple behaviours like non-verbal vocalisation or orientation alternation, and/or could show displacement behaviours rather than simply not performing the task.

In sum, it is important to stress once again that the dog model does not imply that the robot needs to have a dog-like appearance (as a matter of fact we suggest that it should not look like a dog); instead we try to identify simple basic behaviours available to even a mechanical-looking embodiment, such as orientation, proxemic behaviour, gross body movements, comprehending some gestural and verbal communication of the human partner, and being able to show complex and variable repertoire of simple behaviour elements in relevant social situations.

In one of the first application of the above theory we investigated the dynamics of feedback processes during the interaction between a robot used as a hearing aid and human subjects who played the role of the deaf owner (29). The behaviour patterns of the robot, which had no arms and verbal capacities, were designed based on the interactions between hearing dogs and deaf owners. Dog-inspired behaviour sequences and decision making strategies were used to program and control the robot during the trials. Findings indicate that untrained and uninformed participants could correctly interpret the robot's actions, and that head movements and gaze directions during signalling, leading, and feed-back processes were important and effective for communicating the robot's intentions.

\section{Acknowledgement}

The research was supported by the European Union (LIREC-215554) and MTA 01 031. A.M. also received support from the Swiss National Science Foundation (SWARMIX: Synergistic Interactions in Swarms of Heterogeneous Agents).

We would like to thank Barbara Gáspár for her help in conducting the tests. We are grateful to the Hungarian Dogs for Humans charity and the owners for their participation.

\section{References}

1. Amat, J.: Lecture Notes in Control and Information Sciences, Volume 236/1998, 211-221, DOI: 10.1007/BFb 0030807 (1998)

2. Yamazaki, K., Ueda, R., Nozawa, S., Mori, Y., Maki, T., Hatao, N., Okada, Kei., Inaba, M.: "Tidying and Cleaning Rooms by a Daily Assistive Robot -- An Integrated System for Doing Chores in Real World --," Journal of Behavioral Robotics, Volume 1, Number 4, 231-239, (2011)

3. Pollack, M.E., Brown, L., Colbry, D., Orosz, C., Peintner, B., Ramkrishnan, S., Engberg, S., Matthews, J., Dunbar-Jacob, J., McCarthy, C., Thrun, S., Montemerlo, M., Pineau, J., and Roy, N.: "Pearl: A mobile robotic assistant for the elderly,” in AAAI Workshop on Automation as Caregiver (2002) 
4. Bartneck, C., Reichenbach, J., Breemen, A.V.: In Your Face, Robot! The Influence of a Character's Embodiment on How Users Perceive Its Emotional Expressions. Proceedings of Design and Emotion. Ankara. (2004)

5. Miklósi, Á., Gácsi, M.: On the utilization of social animals as a model for social robotics. Frontiers in Psychology, 3, 75. (2012)

6. Dautenhahn K.: Robots We Like to Live With?! - A Developmental Perspective on a Personalized, Life-Long Robot Companion. In Proceedings of the IEEE International Workshop on Robot and Human Interactive Communication (Kurashiki, Okayama, Japan, September 20 - 22, (2004)

7. Jones, T., Lawson, S., Mills, D.: Interaction with a Zoomorphic Robot that Exhibits Canid Mechanisms of Behaviour, Proc of IEEE International Conference on Robotics and Automation (ICRA 2008) Pasadena. (2008)

8. Topál, J., Kubinyi, E., Gácsi, M., Miklósi, Á.: Obeying social rules: A comparative study on dogs and humans. Journal of Cultural and Evolutionary Psychology, 3: 213-239. (2005)

9. Topál, J., Miklósi, Á., Gácsi, M., Dóka, A., Pongrácz, P., Kubinyi, E., Virányi Zs., Csányi, V:: The dog as a model for understanding human social behavior. Advances in the Study of Animal Behaviour, 39: 71-116. (2009)

10. Fallani, G., Prato Previde, E. \& Valsecchi, P: Do disrupted early attachments affect the relationship between guide dogs and blind owners? Applied Animal Behaviour Science 100, 241-257 (2006)

11. Nguyen, H., Kemp, C.C.: Bio-inspired Assistive Robotics: Service Dogs as a Model for Human-Robot Interaction and Mobile Manipulation (2008)

12. Arkin, R.C., Fujita, M., Takagi, T., and Hasegawa, R.: Ethological Modeling and Architecture for an Entertainment Robot, 2001 IEEE International Conference on Robotics and Automation, Seoul, Korea, pp.453-458. (2001)

13. Syrdal, D.S., Koay, K.L., Gácsi, M., Walters, M.L., Dautenhahn, K.: Video Prototyping of Dog-Inspired Non-verbal Affective Communication for an Appearance Constrained Robot. 19th IEEE International Symposium on Robot and Human Interactive Communication, (RO-MAN 10), Viareggio, Italy, pp. 632637 (2010)

14. Hecht, J., Miklósi, Á., Gácsi, M.: Behavioral assessment and owner perceptions of behaviors associated with guilt in dogs. Applied Animal Behaviour Science, 139: 134-142. (2012)

15. Barrett, K.C.: The origins of social emotions and self-regulation in toddlerhood: New evidence, Cognition \& Emotion, 19:7, 953-979. (2005)

16. Maestripieri, D., Schino, G., Aureli, F., Troisi, A.: A modest proposal: displacement activities as an indicator of emotions in primates. Animal Behavior, 44, 967-979. (1992)

17. Miklósi, Á., Pongrácz, P., Lakatos, G., Topál, J., Csányi, V.: A comparative study of the use of visual communicative signals in interactions between dogs (Canis familiaris) and humans and cats (Felis catus) and humans. Journal of Comparative Psychology, 119: 179-186. (2005)

18. Kovács, Sz., Vincze D., Gácsi M., Miklósi Á., Korondi P.: Ethologically inspired robot behavior implementation. 4th International Conference on Human System Interactions Yokohama, Japan. ISSN 2158-2246, ISBN 978-1-4244-9638-9, doi: 10.1109/HSI.2011.5937344) pp. 64-69. (2011) 
19. Numakunai R., Ichikawa T., Gácsi M., Korondi P., Hashimoto H., Niitsuma M.: Exploratory Behavior in Ethologically Inspired Robot Behavioral Model. ROMAN 2012, Paris, France. 577-582. (2012)

20. Faragó, T, Miklósi Á, Korcsok B, Száraz J, Gácsi M Social behaviours in dogowner interactions can serve as a model for designing social robots. IS submitted (2012)

21. Nandy, A., Mondal, S., Prasad, J.S., Chakraborty, P., Nandi, G.C.: Recognizing \& Interpreting Indian Sign Language gesture for Human Robot Interaction. 2010 ICCCT. pp. 712 - 717. (2010)

22. Miklósi, Á., Kubinyi, E., Topál, J., Gácsi, M., Virányi, Zs., Csányi, V.: A simple reason for a big difference: wolves do not look back at humans, but dogs do. Current Biology, Vol. 13, 763-766 (2003)

23. Nickel, K., Stiefelhagen, R.: Visual recognition of pointing gestures for humanrobot interaction, Image and Vision Computing, 25:12, 1875-1884. (2007)

24. Lakatos, G., Gácsi, M., Topál, J., Miklósi, Á.: Comprehension and utilisation of pointing gestures and gazing in dog-human communication in relatively complex situations. Animal Cognition, 15:201-213. (2012)

25. Young, J.E., Kamiyama, Y., Reichenbach, J., Igarashi, T., Sharlin, E.: How to walk a robot: A dog-leash human-robot interface. RO-MAN, IEEE 376 - 382. (2011)

26. Molnár, Cs., Pongrácz, P., Miklósi, Á.: Seeing with ears: Sightless humans' perception of dog bark provides a test for structural rules in vocal communication. The Quarterly Journal of Experimental Psychology, 63: 1004-1013. (2010)

27. Keltner, D.: The signs of appeasement: Evidence for the distinct displays of embarrassment, amusement, and shame. Journal of Personality and Social Psychology, 68, 441-454. (1995)

28. Miller, R. S., \& Leary, M. R.: Social sources and interactive functions of embarrassment. In M. Clark (Ed.), Emotion and social behavior (pp. 322-339). New York: Russell Sage Foundation) (1992)

29. Koay, K.L., Lakatos, G., Syrdal, D.S., Gácsi, M., Bereczky, B., Dautenhahn, K., Miklósi, Á. and Walters, M. L.: Hey! There is someone at your door. A Hearing Robot using Visual Communication Signals of Hearing Dogs to Communicate Intent. submitted SSCI 2013 Alife 